\title{
Articles
}

\section{Ghrelin Arg51Gln mutation is a risk factor for Type 2 diabetes and hypertension in a random sample of middle-aged subjects}

\author{
S. Pöykkö, O. Ukkola, H. Kauma, M. J. Savolainen, Y. A. Kesäniemi \\ Department of Internal Medicine and Biocenter Oulu, University of Oulu, Finland
}

\begin{abstract}
Aims/hypothesis. Experimental studies have suggested that ghrelin, a novel gastrointestinal peptide hormone, could play a role in glucose homeostasis. In addition, ghrelin has been associated with beneficial haemodynamic effects in experimental settings. Since the Arg51Gln mutation changes the carboxyterminal amino acid of the mature hormone and is associated with low ghrelin concentrations, we assessed the hypothesis that Arg51Gln mutation is a risk factor for Type 2 diabetes, impaired glucose tolerance, and hypertension. Methods. Blood pressure recordings and oral glucose tolerance test were carried out in the hypertensive $(n=519)$ and control cohorts $(n=526)$ of our well-defined OPERA study. The genotypes and plasma IGF-I and IGFBP-1 concentrations of 1031 subjects were analysed.
\end{abstract}

Results. The ghrelin $51 \mathrm{Gln}$ allele was a risk factor for Type 2 diabetes, and the effect remained significant after adjustment for age, BMI, and study group $(\mathrm{OR}=2.53$, CI: $1.11-5.75, p=0.027)$. In addition, the $51 \mathrm{Gln}$ allele was a risk factor for hypertension $(\mathrm{OR}=2.63$, CI: 1.37-5.08, $p=0.003)$. 51Gln carriers had lower concentrations of IGF-I and higher concentrations of IGFBP-1 compared to non-carriers.

Conclusion/interpretation. The ghrelin 51Gln allele could increase the risk for Type 2 diabetes and hypertension. The low IGF-I concentrations in 51Gln carriers suggest that the mechanism might be associated with low GH concentrations. [Diabetologia (2003) 46:455-458]

Keywords Type 2 diabetes, hypertension, risk factors, ghrelin, mutation, IGF-I.
Ghrelin is a recently discovered peptide hormone with a very potent GH-releasing effect [1]. It is a somatotrophic, orexigenic and adipogenic hormone, which links the regulatory systems for growth and energy balance [2].

Acute ghrelin administration induces hyperglycaemia and reduces insulin secretion [3] and has beneficial haemodynamic effects in humans [4]. Thus,

Received: 4 October 2002 / Revised: 28 November 2002

Published online: 14 March 2003

C) Springer-Verlag 2003

Corresponding author: S. Pöykkö MSc, Laboratory of Internal Medicine, University of Oulu, P.O. box 5000, 90014 Oulu, Finland

E-mail: seppo.poykko@ppshp.fi

Abbreviations: GH, growth hormone; IGFBP-1, insulin-like growth factor binding protein 1 . changes in the activity or concentration of the hormone might affect insulin metabolism or blood pressure regulation.

The Arg51Gln mutation changes the carboxyterminal amino acid of the mature hormone [5] and is associated with lower ghrelin concentrations [6]. We therefore assessed the hypothesis that the Arg51Gln mutation might be a risk factor for Type 2 diabetes, IGT, and hypertension.

\section{Materials and methods}

Subjects. OPERA (Oulu Project Elucidating Risk of Atherosclerosis) is a population-based epidemiological study addressing the risk factors and disease end-points of atherosclerotic cardiovascular diseases. The study cohort and the selection criteria have been described in detail elsewhere [7]. In short, 600 
hypertensive subjects (300 men and 300 women aged 40-59) were randomly selected from the national register for reimbursement of the costs of antihypertensive medication. For each hypertensive subject, an age- and sex-matched control subject was randomly selected. There were 1045 subjects participating in the study and it was conducted according to the principles of the Declaration of Helsinki.

Biomedical factors and laboratory analyses. At the visit, anthropometric measurements (weight, height, waist, hip), BP measurements, and an OGTT with $75 \mathrm{~g}$ of glucose were carried out. Plasma was separated from venous blood and stored for further analyses. Blood insulin and glucose concentrations were analysed at 0,60 , and $120 \mathrm{~min}$ [7].

Type 2 diabetes and IGT were determined according to the WHO criteria. Fasting plasma IGF-I and IGFBP-1 concentrations were analysed from plasma samples stored at $-20^{\circ} \mathrm{C}$ using commercial kits (DSL-10-2800 ACTIVE Non-Extraction IGF-I ELISA; Diagnostic Systems Laboratories, Webster, Tex., USA and IGFBP-1 IEMA test; Oy Medix Biochemica, Kauniainen, Finland). The routine clinical laboratory tests were carried out in the Central Laboratory of the Oulu University Hospital.

DNA analyses. The 618 bp DNA fragment covering the exons 1 and 2 that encompass the entire ghrelin product was amplified from leukocyte DNA using the PCR technique (forward primer: 5'-GCTGGGCTCCTACCTGAGC-3', reverse primer: 5'-GGACCCTGTTCACTGCCAC-3') [5]. The Arg51Gln mutation was identified using restriction endonuclease $S a c \mathrm{I}$, which retains the mutated site (guanine replaced by adenine) at base 346 in exon 2 of the preproghrelin gene undigested. The nucleotide variant $\mathrm{G} / \mathrm{G}$ corresponds to Arg homozygosity and A/A to Gln homozygosity.

Statistical methods. A $\chi^{2}$-test was carried out to assess whether the observed genotype frequencies were in Hardy-Weinberg equilibrium and whether the prevalence of Type 2 diabetes, hy- pertension, and IGT in the subjects with and without mutation differed from each other. To compare the means of the variables measured, a Student's $t$ test, analysis of covariance (ANCOVA), and Mann-Whitney's U-test were used. In the multivariate analyses, BMI, age, study group (when appropriate), and Arg51Gln genotype were added as explanatory factors. The one $51 \mathrm{Gln}$ homozygote identified was pooled among the heterozygotes in the analyses. The three subjects found to have Type 1 diabetes were excluded from this study.

To normalize the skewed distributions of IGF-I, IGFBP-1, triglycerides, HDL cholesterol, glucose, and insulin, log-transformed values were used as appropriate. Results with $p$-value less than 0.05 were considered statistically significant. All calculations were made with the SPSS (version 9.0; SPSS, Inc.) statistical package. All the tests carried out were two-sided.

\section{Results}

The allele frequency of ghrelin $51 \mathrm{Gln}$ was $2.2 \%$ in the whole data set. The main characteristics of the study subjects by ghrelin Arg51Gln genotypes are shown (Table 1). In the pooled data, 51Gln carriers had lower concentrations of IGF-I $(p=0.036)$ but higher concentrations of IGFBP-1 $(p=0.033)$. When adjusted for the effect of age, BMI, sex, and study group, 51Gln remained a predictor of both IGF-1 concentrations $(p=0.023)$ and IGFBP-1 concentrations $(p=0.048)$. In the control cohort, the $51 \mathrm{Gln}$ allele was associated with lower AUC insulin $(p=0.039)$, whereas in the hypertensive cohort, it was associated with higher IGFBP-1 ( $p=0.033$, after adjustment $p=0.054$ ). The only subject identified as homozygous for $51 \mathrm{Gln}$ was obese $(\mathrm{BMI}=31.1)$ and quite short $(151 \mathrm{~cm})$ and had low IGF-I $(66 \mathrm{ng} / \mathrm{ml})$ and high $\mathrm{HbA}_{1 \mathrm{c}}$ concentrations $(8.4 \%)$.

Table 1. Main characteristics of the study subjects by ghrelin Arg51Gln genotype

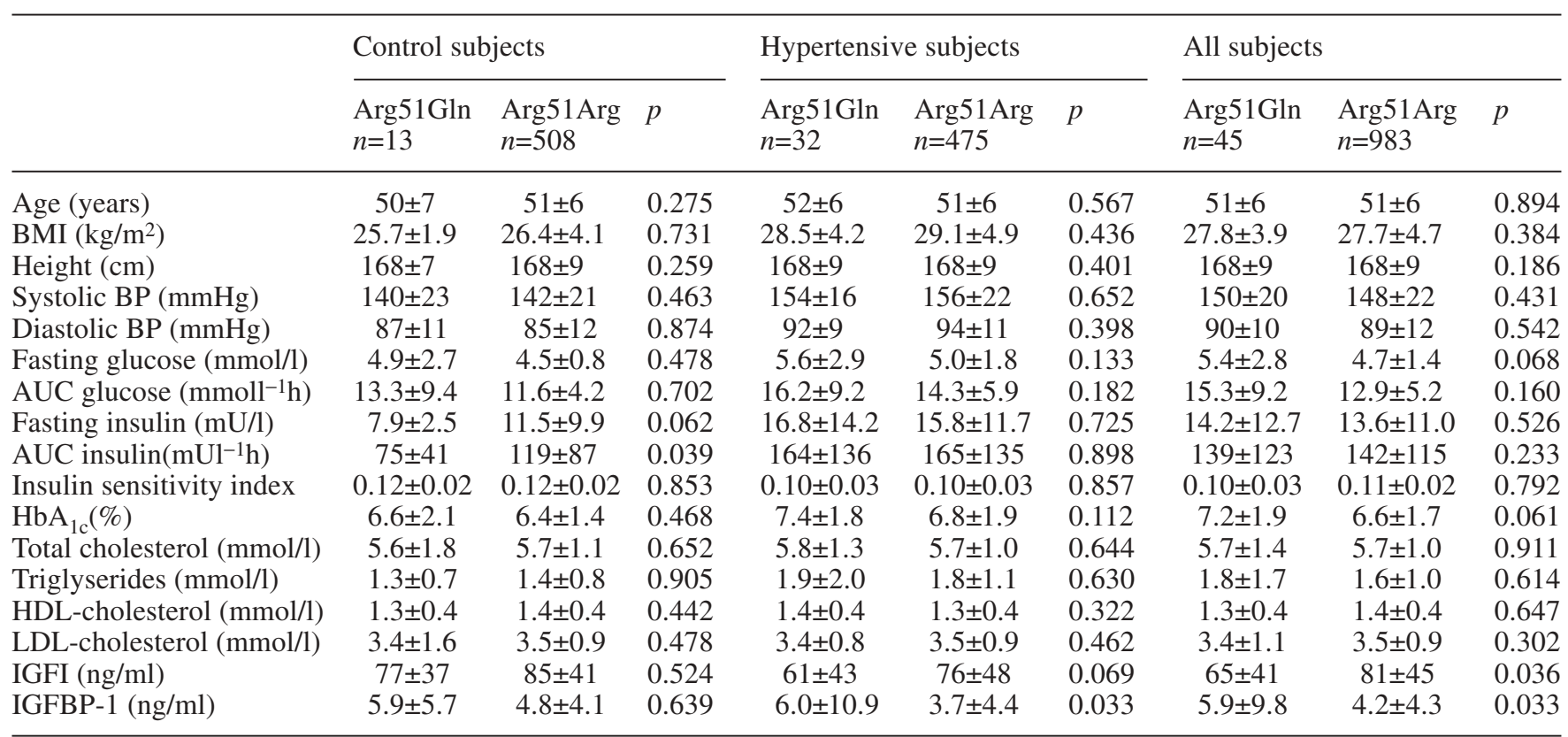

Data are means \pm SD. $\mathrm{p}=$ probability for the difference between the genotypes in ANCOVA adjusted for sex (control subjects and hypertensive subjects) or sex and study group (all subjects). Gln51Gln subject was pooled to the heterozygotes in the analyses. 


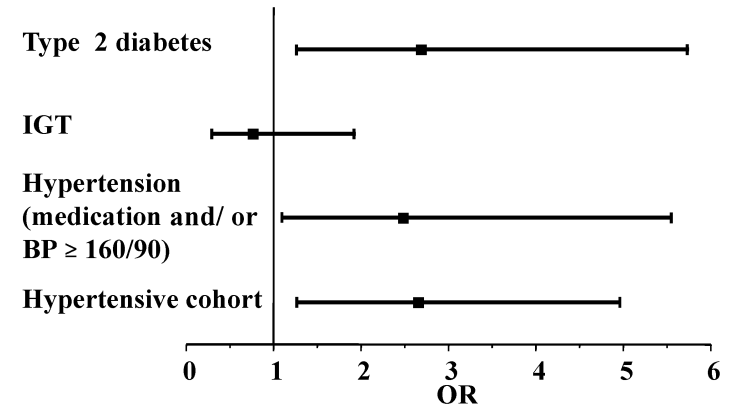

Fig. 1. Odds ratios with $95 \% \mathrm{CI}$ of the ghrelin $51 \mathrm{Gln}$ allele for Type 2 diabetes, IGT and hypertension in the pooled data obtained by $\chi^{2}$-test

We show the odds ratios with $95 \% \mathrm{CI}$ of the ghrelin $51 \mathrm{Gln}$ allele for Type 2 diabetes, IGT, and hypertension (Fig. 1). In the pooled data, the 51 Gln allele was a risk factor for Type 2 diabetes $\left(\chi^{2}=6.86, \mathrm{df}=1\right.$, $p=0.009$, OR=2.67). The effect of the $51 \mathrm{Gln}$ allele as a risk factor for Type 2 diabetes was also evident in the logistic regression model of the pooled data $(\mathrm{OR}=2.53$, CI: $1.11-5.75, p=0.027)$ or only the hypertensive cohort $(\mathrm{OR}=2.49, \mathrm{CI}: 1.02-6.07, p=0.044)$. The allele frequencies in subjects with and without Type 2 diabetes were $4.8 \%$ and $2.0 \%$, respectively. However, the Arg51Gln mutation was not associated with the prevalence of IGT $\left(\chi^{2}=0.30, \mathrm{df}=1, p=0.585\right)$.

The $51 \mathrm{Gln}$ allele was more common in the hypertensive $(3.1 \%)$ than in the control cohort $(1.3 \%)$ $\left(\chi^{2}=8.94, \mathrm{df}=1, \mathrm{OR}=2.63, \mathrm{CI}: 1.37-5.08, p=0.003\right)$ (Fig. 1). The difference remained significant when all hypertensives (subjects of the hypertensive cohort and the subjects on antihypertensive medication and/or with $\mathrm{BP} \geq 160 / 90$ in the control cohort) were compared to non-hypertensives (no antihypertensive medication and $\mathrm{BP}<160 / 90)\left(\chi^{2}=4.98, \mathrm{df}=1, \mathrm{OR}=2.46, \mathrm{CI}: 1.09\right.$ $5.59, p=0.026)$. Among the hypertensive subjects, $51 \mathrm{Gln}$ carriers had lower IGF-I $(p=0.023)$ and higher IGFBP-1 concentrations $(p=0.016)$ than non-carriers after adjustment for age, BMI, and sex. The mean values of systolic and diastolic BP between 51Gln carriers and non-carriers did not differ from each other.

The Arg51Gln mutation had no effect on the crude mean values of glucose and insulin in either normal subjects or in subjects with Type 2 diabetes or IGT. However, after adjustment for age, BMI, sex, glycaemic status, and diabetes medication, the $51 \mathrm{Gln}$ allele was a predictor of the 2-h plasma glucose concentration in OGTT $(p=0.011)$.

\section{Discussion}

The amino acid Arg51 is a target site for endoprotease action, which leads to proteolytic cleavage of the carboxy-terminal 66 amino acids to produce mature ghrelin. The Arg51Gln mutation disrupts the recognition site in the last codon of the mature ghrelin prod- uct [5]. Interestingly, the mutation was associated with lower IGF-I concentrations which, in most conditions, reflect the GH concentrations quite well [8]. This is in accordance with the earlier report showing an association between the Arg51Gln mutation and low plasma ghrelin concentrations [6].

In this study the $51 \mathrm{Gln}$ allele frequency was almost three-fold in the patients with Type 2 diabetes compared with those without the disease. In addition, the $51 \mathrm{Gln}$ allele was a predictor of a high 2-h glucose value in OGTT. The frequency of the Arg51Gln mutation is quite low and at the population level it is able to explain the increased risk for Type 2 diabetes only marginally. However, the mutation seems to have obvious consequences for the affected individuals, though they represent only a small fraction of the Type 2 diabetic subjects. Since ghrelin is an orexigenic and adipogenic hormone, low ghrelin concentrations could be protective against Type 2 diabetes. However, it seems that obese subjects with Type 2 diabetes have lower ghrelin concentrations than control subjects [9]. This is in accordance with our results, which indicate that Type 2 diabetes is more common amongst 51Gln carriers with low ghrelin concentrations.

Ghrelin has been shown to stimulate insulin secretion in in vivo experiments [10]. In contrast, a recent observation showed that acute administration of ghrelin to humans causes hyperglycaemia and decreases insulin secretion [3]. Thus, the definite role of ghrelin in glucose and insulin metabolism is unclear. As far as the effect of the Arg51Gln mutation on glucose metabolism is concerned, one could speculate that insulin secretion in 51Gln allele carriers could only partially compensate for the diminished insulin action, leading to hyperglycaemia and eventually to Type 2 diabetes.

Ghrelin, when administered intravenously to healthy volunteers, exerts beneficial haemodynamic effects by reducing mean arterial pressure and increasing cardiac output without increasing heart rate [4]. Therefore, a low plasma ghrelin concentration could be expected to be haemodynamically harmful. This notion is supported by these findings, which showed the frequency of hypertension to be lower among $51 \mathrm{Gln}$ non-carriers than among carriers who had earlier been reported to have lower plasma ghrelin concentrations [6]. One potential mechanism behind the hypertensive effect of the 51Gln allele might be associated with the lower IGF-I concentration of 51Gln carriers, since IGF-I has been reported to stimulate endothelial nitric oxide formation [11].

The ghrelin gene has been mapped to the chromosomal region 3p26-p25. Linkage disequilibrium with genes close to the ghrelin gene might explain the association between the ghrelin 51Gln allele and Type 2 diabetes and hypertension. However, no potential candidate genes for Type 2 diabetes or hypertension close enough to the ghrelin gene have been found. 
In conclusion, our findings suggest that the ghrelin 51Gln allele might be a novel risk factor for Type 2 diabetes and hypertension in middle-aged subjects. The low IGF-I concentrations in 51Gln carriers suggest that the mechanism might be associated with low GH concentrations.

Acknowledgements. This study was supported by the Medical Council of the Academy of Finland and the Finnish Foundation for Cardiovascular Research. We acknowledge the excellent technical assistance of Ms H. Häikiö, Ms H. Kalliokoski, Ms S. Kortetjärvi, Ms S. Rannikko, Ms E. Saarikoski and Ms R. Vanhanen.

\section{References}

1. Arvat E, Di Vito L, Broglio F et al. (2000) Preliminary evidence that Ghrelin, the natural GH secretagogue (GHS)receptor ligand, strongly stimulates $\mathrm{GH}$ secretion in humans. J Endocrinol Invest 23:493-495

2. Ukkola O, Pöykkö S (2002) Ghrelin, growth and obesity. Ann Med 34:102-108

3. Broglio F, Arvat E, Benso A et al. (2001) Ghrelin, a natural GH secretagogue produced by the stomach, induces hyperglycemia and reduces insulin secretion in humans. J Clin Endocrinol Metab 86:5083-5086
4. Nagaya N, Kojima M, Uematsu M et al. (2001) Hemodynamic and hormonal effects of human ghrelin in healthy volunteers. Am J Physiol Regul Integr Comp Physiol 280:R1483-R1487

5. Ukkola O, Ravussin E, Jacobson P et al. (2001) Mutations in the preproghrelin/ghrelin gene associated with obesity in humans. J Clin Endocrinol Metab 86:3996-3999

6. Ukkola O, Ravussin E, Jacobson P et al. (2002) Role of ghrelin polymorphisms in obesity based on three different studies. Obes Res 10:782-791

7. Kiema TR, Kauma H, Rantala AO et al. (1996) Variation at the angiotensin-converting enzyme gene and angiotensinogen gene loci in relation to blood pressure. Hypertension 28:1070-1075

8. Le Roith D, Scavo L, Butler A (2001) What is the role of circulating IGF-I? Trends Endocrinol Metab 12:48-52

9. Shiiya T, Nakazato M, Mizuta M et al. (2002) Plasma ghrelin levels in lean and obese humans and the effect of glucose on ghrelin secretion. J Clin Endocrinol Metab 87:240-244

10. Date Y, Nakazato M, Hashiguchi S et al. (2002) Ghrelin is present in pancreatic alpha-cells of humans and rats and stimulates insulin secretion. Diabetes 51:124-129

11. Boger RH, Skamira C, Bode-Boger SM, Brabant G, von Zur MA, Frolich JC (1996) Nitric oxide may mediate the hemodynamic effects of recombinant growth hormone in patients with acquired growth hormone deficiency. A double-blind, placebo-controlled study. J Clin Invest 98:2706-2713 